\title{
Cobalt Nano Particle Size Dependence of Noise Modulations in Relation to Nonlinearity*
}

\author{
Toshio Kawahara ${ }^{\dagger}$ and Satarou Yamaguchi \\ Center of Applied Superconductivity and Sustainable Energy Research, \\ Chubu University, 1200 Matsumoto, Aichi 487-8501 Japan
}

\author{
Kenzo Maehashi, Yasuhide Ohno, Kazuhiko Matsumoto, and Tomoji Kawai \\ The Institute of Scientific and Industrial Research, \\ Osaka University, 8-1 Mihogaoka, Osaka 567-0047 Japan \\ (Received 1 September 2009; Accepted 1 February 2010; Published 13 March 2010)
}

\begin{abstract}
Carbon nanotubes (CNTs) are low-dimensional materials, and their surface therefore affects several properties of CNTs. For example, CNT field effect transistors (CNTFETs) are well known as noisy devices caused by the adhesion of molecules on the surface. However, nonlinear systems sometimes have advantages in working with noise. Therefore, we combined the noise CNTFET and another transistor of CNT for a trial nonlinear system. The sine wave amplification in the transistor with noise was then measured. In this system, the control of noise is required for operation of the device and this might be common for nano-sized devices. We can control the noise properties of CNTFET through the control of the Co nano particles catalyst. Depending on fabrication conditions, the noise of a CNTFET has different noise intensity and gate voltage $\left(V_{\mathrm{g}}\right)$ dependence with $1 / f$ type noise. Noise in CNTFETs grown on small Co particles can be controlled within the small $\left|V_{\mathrm{g}}\right|$, and the control range for noise is wide. We studied the modification of nonlinearity using our trial nonlinear system. It was governed by the ratio of the signals and the noise, and shows the robustness of modification. [DOI: 10.1380/ejssnt.2010.115]
\end{abstract}

Keywords: Carbon nanotube; Cobalt; Nano-particles; Electron microscopy; Electrical transport measurements

\section{INTRODUCTION}

Low-dimensional materials are highly attractive because of their novel properties. Among them, carbon nanotubes (CNTs) $[1,2]$ are very promising materials for nano structure building blocks and nano devices. Many applications have been proposed such as nano-size transistors, bio sensors, and interconnections in large-scale circuits. Several technologies can be utilized in these applications, such as high-sensitive gas sensors [3], label-free detection for bio molecules $[4,5]$, and n-type field effect transistor (FET) fabrication [6, 7]. The room temperature operation of single electron transistors, which can usually work only at low temperature, can be archived through the use of nanotechnology [8, 9].

For actual applications, the control of CNT growth through catalyst control seems to be one of the important technologies [10]. The CNT has very specific properties that depend on the rolling properties such as diameter and chirality [11]. Aspect of carbon nanotube field effect transistors (CNTFETs) such as diameter and alignment of channels can also be controlled by the nano fabrication of the catalyst. Cobalt or iron are frequently used catalyst materials for single-walled CNTs (SWCNTs). They can work as catalysts for CNT growth with methane for the forms of SWCNT or their bundles depending on the growth conditions [12]. Carbon complexes of iron or cobalt catalyst during CNT growth have also been studied with electron microscopy to observe the detailed growth mechanism $[13,14]$. We can also use thin film or nano

\footnotetext{
*This paper was presented at 10th International Conference on Atomically Controlled Surfaces, Interfaces and Nanostructures (ACSIN-10), Granada Conference Centre, Spain, 21-25 September, 2009 .

†Corresponding author: toshi@isc.chubu.ac.jp
}

particles for the catalyst. Nano particles seem to be better in terms of controllability. Co nano particles can also be used as an effective catalyst for SWCNTs and the arc plasma gun (APG) is better for the control of the size of deposited materials [15]. Therefore, we used APG for the control of Co nano particles as catalyst for SWCNTs.

As one of the applications of growth control, we constructed model nonlinear systems using CNTFETs. When the nonlinear systems work with noise, the noise may sometimes have constructive roles depending on the noise conditions, such as those associated with stochastic resonance (SR) [16]. SR was first proposed for ice age periodicity by Nicolis [17]. Under a small change of the solar power irradiating the earth, he proposed that an ice age transition can occur with the help of the environmental noise of the earth. Many SR phenomena have been reported for biological applications [18] such as the small signal detection of crayfish [19], information gain in ion channels [20], signal processing in the human brain [21] and so forth.

Artificial devices that have exhibited SR phenomena were also fabricated using CNT [22] or nanowires [23]. For these applications, CNTs or nanowires were used only as transistors with a suitable threshold level, and the noise was applied from outside the systems. However, CNTs were known as noisy devices [24, 25]. Nonlinear devices combined with the noise devices could then be constructed on a single chip. Such a CNTFET nonlinear system seems to be very useful as a model for nano devices. For example, the new working condition for complementary metal oxide semiconductor (CMOS) has recently been studied based on SR phenomena [26].

There are many discussions regarding the origins of noise in CNTs [27]. However, it is resistive noise and can be controlled by the carrier number [28]. Therefore, for the control of noise devices, we can modify the noise intensity by the gate voltage $\left(V_{\mathrm{g}}\right)$ of CNTFETs with the 


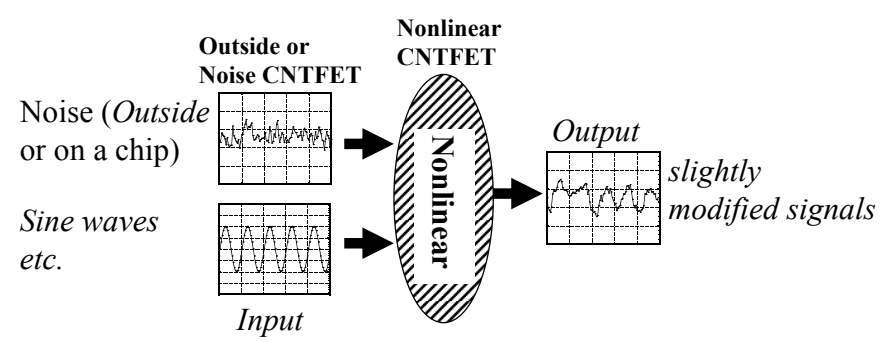

FIG. 1: Nonlinear system working with noise. Noise CNTFET generates noise in our model systems.

carrier number control of CNT channels by the fabrication conditions. Thus, the wide control range of noise intensity through catalyst control is possible for the noise CNTFETs in nonlinear systems.

In this paper, we used Co nano particles as the catalyst for CNT growth, where the nano particle size could be controlled by APG. We studied the size of nano particles, using transmission electron microscopy (TEM) and its dependence on the deposition amounts of Co. The growth of CNTs using these nano particles was also studied and CNTFETs were fabricated. We combined the noise CNTFET and another transistor of CNT for the model nonlinear system. Conventional nonlinear systems which have SR effects are schematically shown in Fig. 1, where noise comes from the outside. In contrast, the noise in our model nonlinear system can be generated by noise CNTFET, which could be controlled by $V_{\mathrm{g}}$. It would be promising to integrate them on a single chip in the future. For noise CNTFETs, we discuss $V_{\mathrm{g}}$ control of the noise intensity depending on the catalyst sizes. The sine wave amplification on the nonlinear CNTFET with noise was then measured.

\section{EXPERIMENTAL}

We used APG for the growth of Co nano particles which were used as catalysts for SWCNT growth. APG is a co-axial arc evaporation gun, and arc discharge in vacuum can generate the plasma of cathode material that is deposited on the substrate $[29,30]$. The deposited material easily forms nano particles. Cathode voltage was $100 \mathrm{~V}$ and charging capacitance was $8800 \mu \mathrm{F}$. The deposition amount can be controlled by the shot pulse number of APG and is expressed by the equivalent thickness to the film thickness by the same amount. These values can be calibrated by the thick film, where the APG-grown materials also form the thin film whose thickness can be measured with the profilometer. We used two different amounts for the Co catalysts. For sample 1, The equivalent thickness of Co was $4 \mathrm{~nm}$ for sample 1 and $0.4 \mathrm{~nm}$ for sample 2. We also used electron beam (EB) deposited thin films as a catalyst for sample 3. Using these Co catalysts, the CNTFETs were fabricated with the alcohol CVD, where the deposition temperature was $820^{\circ} \mathrm{C}$ and deposition time was $10 \mathrm{~min}$. The backgate-type FET structure was made by conventional lithography on a $\mathrm{Si}$ wafer, where the thickness of the $\mathrm{SiO}_{2}$ layer was $500 \mathrm{~nm}$. $\mathrm{Ti}(1 \mathrm{~nm}) / \mathrm{Pd}(25 \mathrm{~nm})$ electrodes were used for the drain and source terminals as ohmic contacts. The distance for drain and source electrodes was $2.5 \mu \mathrm{m}$ and the width of electrodes was $17 \mu \mathrm{m}$, where the channel length was slightly longer than this distance depending on the growth direction. We used atomic force microscope (AFM) to observe for the CNTFET channels as shown in Fig. 2. The channels length was about $3 \mu \mathrm{m}$ for sample 1 and $4 \mu \mathrm{m}$ for sample 2. The channels diameter was also measured by $\mathrm{AFM}$ as their height, and it was $1.06 \pm 0.20 \mathrm{~nm}$ for sample 1 and $0.78 \pm 0.18 \mathrm{~nm}$ for sample 2 .

(a)

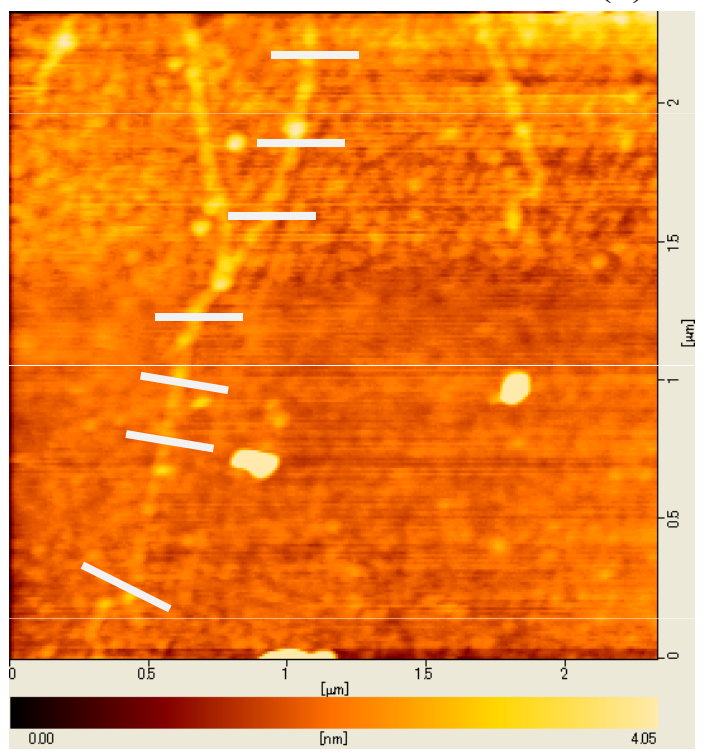

(b)

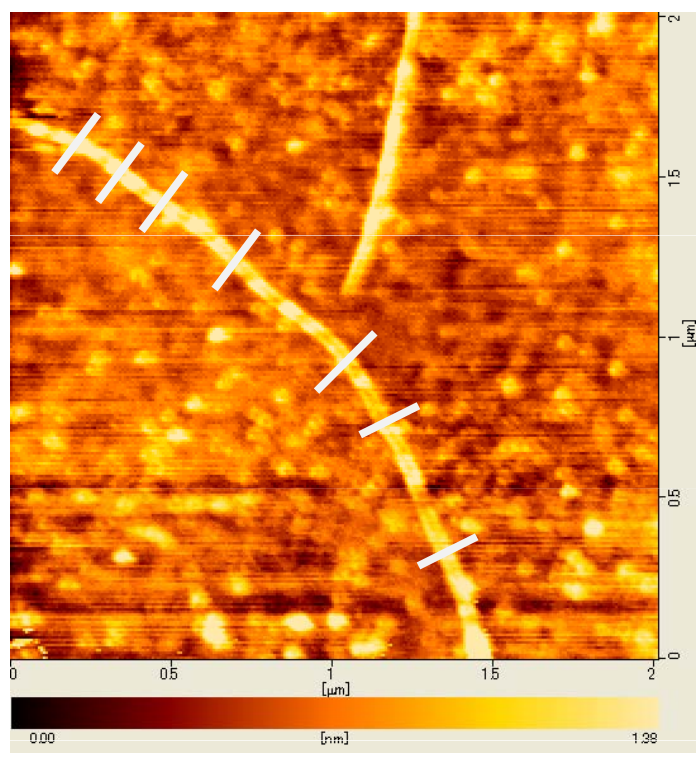

FIG. 2: Representative AFM images for samples 1 (a) and 2 (b). The white lines express the measured points for the channel height.

We observed Co nano particles on a $\mathrm{SiO}_{2}$ membrane by TEM. For the TEM samples, a thin substrate of $\mathrm{SiO}_{2}$ was deposited by $\mathrm{EB}$ evaporation on a $\mathrm{NaCl}$ crystal followed by Co nano particle deposition. The lift-off samples were used for TEM observations, where the thickness of a substrate was optimized depending on the deposition 
conditions of catalysts. High-resolution TEM images were obtained with an acceleration voltage of $300 \mathrm{kV}$.

FET properties of CNTFETs were measured by the semiconductor device analyzer B1500 (Agilent Technologies). Current-voltage ( $I V)$ characteristics were measured from $-1 \mathrm{~V}$ to $1 \mathrm{~V}$ of $V_{D S}$ with $V_{\mathrm{g}}$ scanned from $-5 \mathrm{~V}$ to $5 \mathrm{~V}$ with a step of $1 \mathrm{~V}$. $V_{\mathrm{g}}$ dependence of $I_{\mathrm{DS}}$ was also measured. Noise power spectral density (PSD) was calculated from the time dependence of $I_{\mathrm{DS}}$ with constant $V_{\mathrm{DS}}$ and $V_{\mathrm{g}}$, where the fast Fourier transform (FFT) analysis with the Bartlett windows function was used. For measurements of $V_{\mathrm{g}}$ dependence of noise intensity, we scanned $V_{\mathrm{g}}$ between $-5 \mathrm{~V}$ and $-0.5 \mathrm{~V}$.

We used a combination of two CNTFETs for the model nonlinear systems. One is the noise CNTFET that is sample 1 or sample 2, which can generate noise. Another is the nonlinear CNTFET of sample 3, where the sum of the noise and sine waves was fed into $V_{\mathrm{g}}$ and output $I_{\mathrm{DS}}$ was used for the nonlinear response estimation. The detailed conditions of input signals are presented in the next section. Sample 3 has a large $I_{\mathrm{DS}}$ and then it seems to fit for the nonlinear CNTFET. For the nonlinear response estimation, we used the PSD of $I_{\mathrm{DS}}$ of the nonlinear CNTFET, where averaged data taken over 4 times under the same conditions were used for the statistical analysis. PSD was also calculated from the time dependence of $I_{\mathrm{DS}}$ by FFT.

\section{RESULTS AND DISCUSSION}

At first, we will discuss the TEM images of Co nano particles on a $\mathrm{SiO}_{2}$ membrane. In Fig. 3 (a), Co nano particles for sample 1 are shown and large nano particles can be seen with sizes between $4 \mathrm{~nm}$ and $6 \mathrm{~nm}$. In Comparison, Fig. 3 (b) shows no large particles and their sizes range from $3 \mathrm{~nm}$ to $4 \mathrm{~nm}$. These nano particles correspond to the catalyst for sample 2 . Thus, we can control Co nano particle size by the deposition amount. On the $\mathrm{SiO}_{2}$ substrate for the devices, smaller nano particles could be deposited because of the strong interaction with the substrate. These Co nano particles effectively work as a catalyst, and many CNTs could be grown on the smaller nano particles with the same conditions of sample 2, when CNTs were grown on a Si wafer to check the growth condition. The suitable size of Co nano particles, of course, can act as the catalyst for CNT growth and the smaller size of particles seems to be better for our systems.

Next, we investigate the $I V$ characteristics for noise CNTFETs in Fig. 4. Figure 4 (a) is for sample 1 and the current should be suppressed at the larger $V_{\mathrm{g}}$ because of $p$-type carriers. In Fig. 4 (b), the $I V$ characteristics for sample 2 are plotted. In Comparison with sample 1 , the current of sample 2 becomes small at the same $V_{\mathrm{g}}$ of sample 1 , where conductances at $V_{\mathrm{g}}=-5 \mathrm{~V}$ are $1.2 \times 10^{-7} \mathrm{~S}$ for sample 1 and $6.8 \times 10^{-8} \mathrm{~S}$ for sample 2 . As shown in Fig. 5, $V_{\mathrm{g}}-I_{\mathrm{DS}}$ curves also support the small conductance for sample 2. Conductances at $V_{\mathrm{g}}=-5 \mathrm{~V}$ for several CNTFETs on the same substrate are listed in Table I. The tendency of small conductance could be seen for the CNTFETs with small amount of Co as sample 2. The difference in the channel length is about $25 \%$, then one of the contributions on conductance difference seems to be the
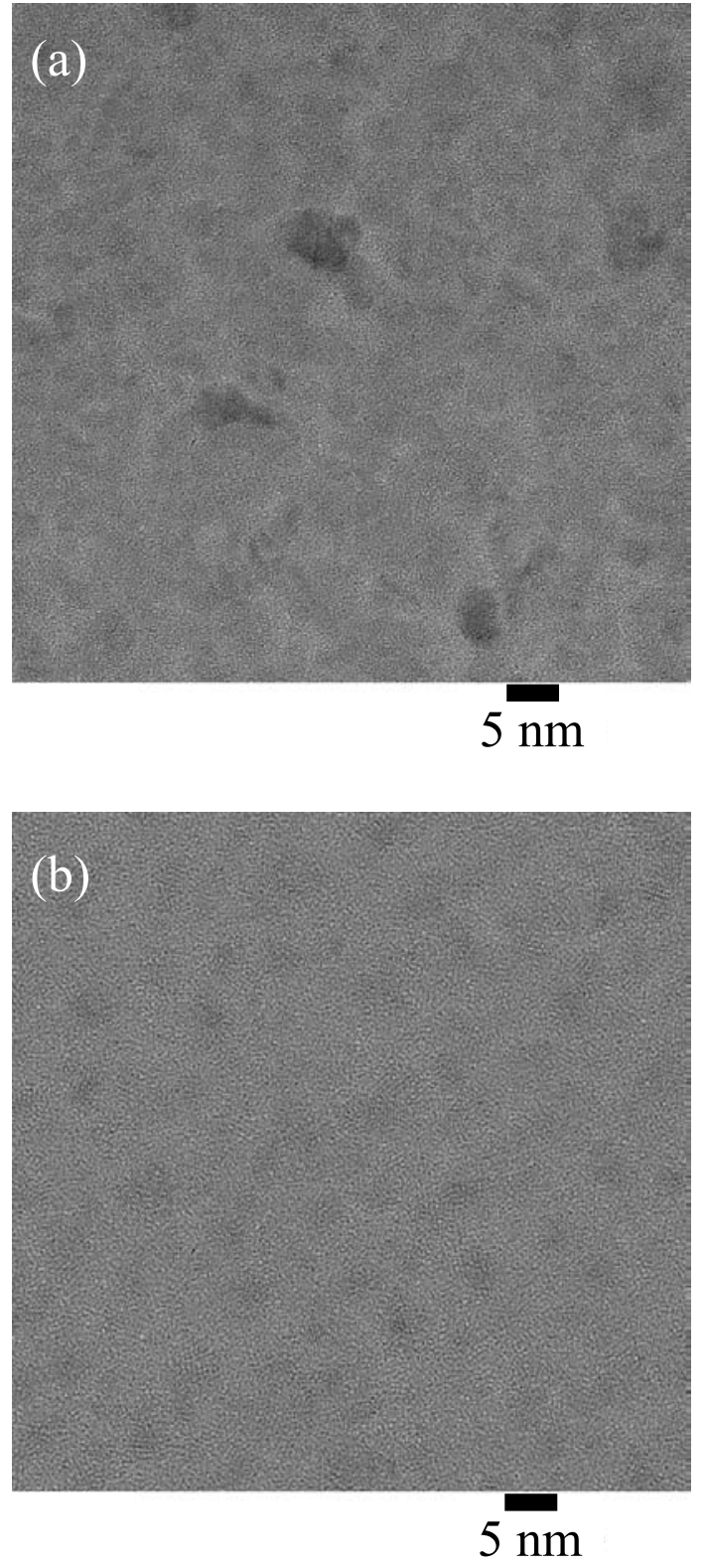

FIG. 3: TEM images for Co nano particles on a $\mathrm{SiO}_{2}$ membrane. In (a) and (b), the equivalent thickness of deposited Co is $4 \mathrm{~nm}$ and $0.4 \mathrm{~nm}$, respectively.

diameter difference of channels. It could be also caused by defects in channels or contact resistance on electrodes. The asymmetric $I V$ characteristics could be caused by an interfacial potential between electrodes and a CNT with the degradation of electrodes, as oxidization of $\mathrm{Ti}$ buffer layers could asymmetrically degrade drain and/or source electrodes. However, these electrode problems might affect $V_{\mathrm{g}}$ dependence of noise intensity only at larger $V_{\mathrm{g}}$.

CNTFETs have large noise of a $1 / f$ type. The PSD with constant $V_{\mathrm{g}}$ of CNTFET is plotted in Fig. 6 (a) for sample 1. Sample 2 also has a $1 / f$ type noise. The noise in CNTFET can be controlled by the carrier number, and its intensity should then depend on $V_{\mathrm{g}}$. Therefore, we plotted the $V_{\mathrm{g}}$ dependence of the noise intensity, where the PSD at $5 \mathrm{~Hz}$ is plotted as shown in Figs. 6 (b) and (c) for sample 1 and sample 2, respectively. Several FETs on the same chip have similar noise intensity and we selected one 
(a)

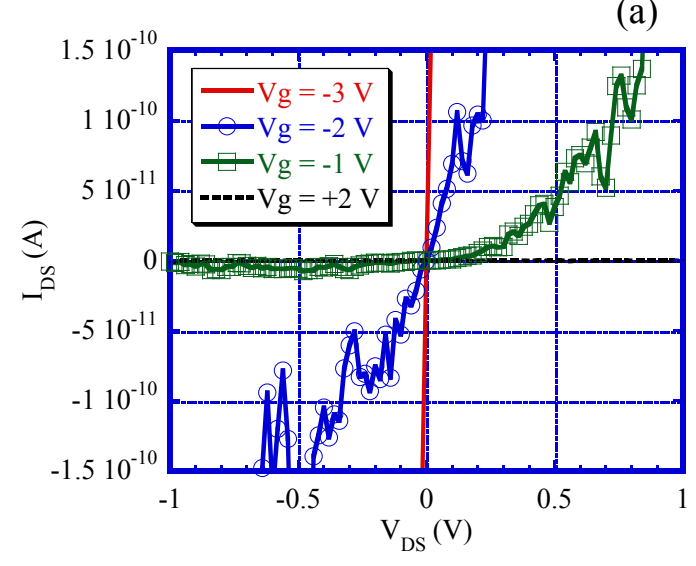

(b)

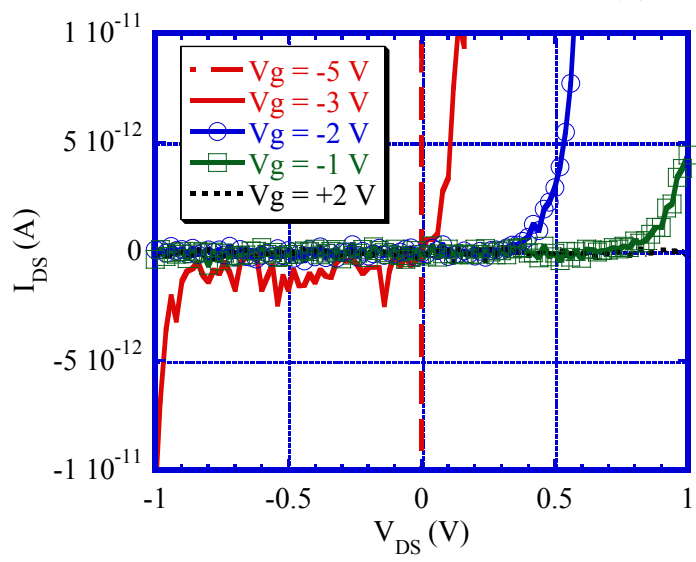

FIG. 4: Representative current-voltage characteristics for CNTFETs used as noise devices. (a) Samples 1, (b) Sample 2. Solid lines: $V_{\mathrm{g}}=-3 \mathrm{~V}$; solid lines with circles: $V_{\mathrm{g}}=-2 \mathrm{~V}$; solid lines with squares: $V_{\mathrm{g}}=-1 \mathrm{~V}$; dotted lines: $V_{\mathrm{g}}=+2 \mathrm{~V}$. In (b), the dashed line is for $V_{\mathrm{g}}=-5 \mathrm{~V}$.

for nonlinear response measurements. However, PSDs are normalized by $I_{\mathrm{DS}}^{2}$ for the comparison of FETs on the different chips. The noise PSD drastically changed between $V_{\mathrm{g}}=-2 \mathrm{~V}$ and $0 \mathrm{~V}$ for sample 1 , and changed slightly between $V_{\mathrm{g}}=-2 \mathrm{~V}$ and $-5 \mathrm{~V}$. Thus, at the small $\left|V_{\mathrm{g}}\right|$ below $|2| \mathrm{V}$, noise intensity can be widely controlled. For sample 2 , the noise intensity slightly increases at a $V_{\mathrm{g}}$ of $-4 \mathrm{~V}$

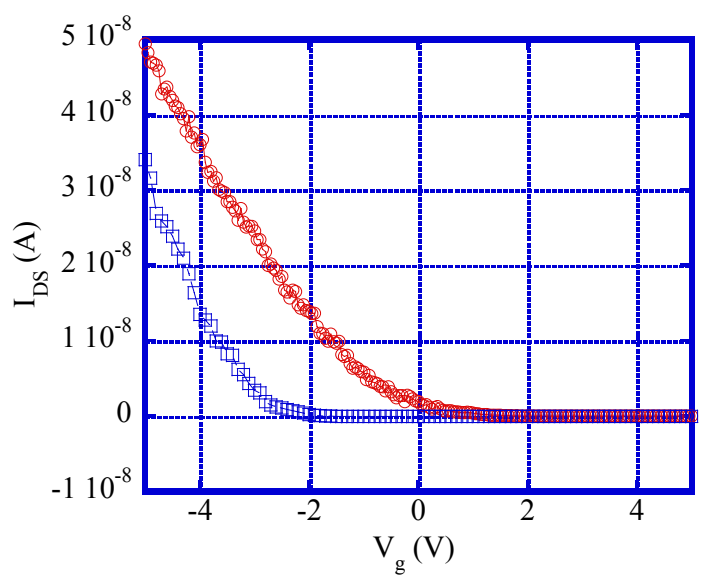

FIG. 5: $V_{\mathrm{g}}$ dependence of $I_{\mathrm{DS}}$ for samples 1 (circles) and 2 (squares) with $V_{\mathrm{DS}}$ of $0.2 \mathrm{~V}$.
TABLE I: Representative conductance of CNTFETs on each chip at $V_{\mathrm{g}}=-5 \mathrm{~V}$.

\begin{tabular}{lc}
\hline \hline Sample $1^{a}$ & Sample 2 \\
\hline $3.4 \times 10^{-7} \mathrm{~S}$ & $6.8 \times 10^{-8} \mathrm{~S}$ \\
$3.3 \times 10^{-7} \mathrm{~S}$ & $6.7 \times 10^{-8} \mathrm{~S}$ \\
$2.5 \times 10^{-7} \mathrm{~S}$ & $4.0 \times 10^{-8} \mathrm{~S}$ \\
$1.2 \times 10^{-7} \mathrm{~S}$ & $8.3 \times 10^{-8} \mathrm{~S}$ \\
\hline \hline
\end{tabular}

${ }^{a}$ Note: It means that this column summarizes conductance for the CNTFETs on the same substrate for sample 1.

and $-5 \mathrm{~V}$ because of the contact noise at the electrode. Nevertheless, $V_{\mathrm{g}}$ dependence is almost the same as that of sample 1 between $V_{\mathrm{g}}=-2 \mathrm{~V}$ and $-5 \mathrm{~V}$. On the other hand, at the small $\left|V_{\mathrm{g}}\right|$ below $|2| \mathrm{V}$, the change of noise intensity on sample 2 by $V_{\mathrm{g}}$ is larger than that on sample 1. This means that total carrier number in the CNT channel is small. Therefore, a small Co catalyst could generate small diameter CNTs and/or defective ones. It is possible that defects hardly affect the electric properties of a smaller-diameter CNT because of the large ratio of defective area to small surface area. In these cases, resistance of channels might be large. We are now trying noise spectroscopy for further investigation. On the other hand, the $V_{\mathrm{g}}$ dependence indicates that sample 2 seems to be better for noise devices that have controllability with the wide range.

Finally, we show the effects of these noises on the nonlinear properties of CNTFETs. We construct model nonlinear systems to combine two CNTFETs of noise CNTFET and nonlinear CNTFET as shown in Fig. 1. The noise signals from the noise FET were added on the sine waves using the function generator, and were fed into sample 3. The averaged signal level was set just above the sub-threshold voltage of nonlinearity on sample 3. Here we set the averaged values of input signals to $0.8 \mathrm{~V}$. The noise intensity is expressed by the normalized noise intensity, where unity expresses the noise intensity of $1.1 \mathrm{~V}$ as the peak-to-peak voltage on $V_{\mathrm{g}}$ in relation to the time dependence.

The nonlinear response is estimated by the correlation between the input and output signals on the nonlinear system. There are several methods for the estimation such as FFT analysis for the output signals [16], cross correlation between input and output signals [31, 32], and information entropy for the output signals [33, 34]. For aperiodic signals, cross correlation is better, and information analysis fits for very noisy signals [35]. However, we used large input signals above sub-threshold levels because we would like to observe the modification of nonlinearity [36, 37] for our model systems. Additionally, we used periodic signals for the trial input signals for simplicity. Therefore, we used PSD of output $I_{\mathrm{DS}}$ as the estimation for the nonlinear response. The peak intensity of the output signals at $5 \mathrm{~Hz}$ that is the input frequency is calculated as the difference between the PSD at $5 \mathrm{~Hz}$ and the background intensity estimated by the linear interpolation.

These peak intensities are plotted in Figs. 7 (a) and (b) as a function of the normalized noise intensity for sample 1 and sample 2, respectively. In both noise dependencies, the enhancement of the output signals in comparison to the small noise case can be clearly observed. The noise 

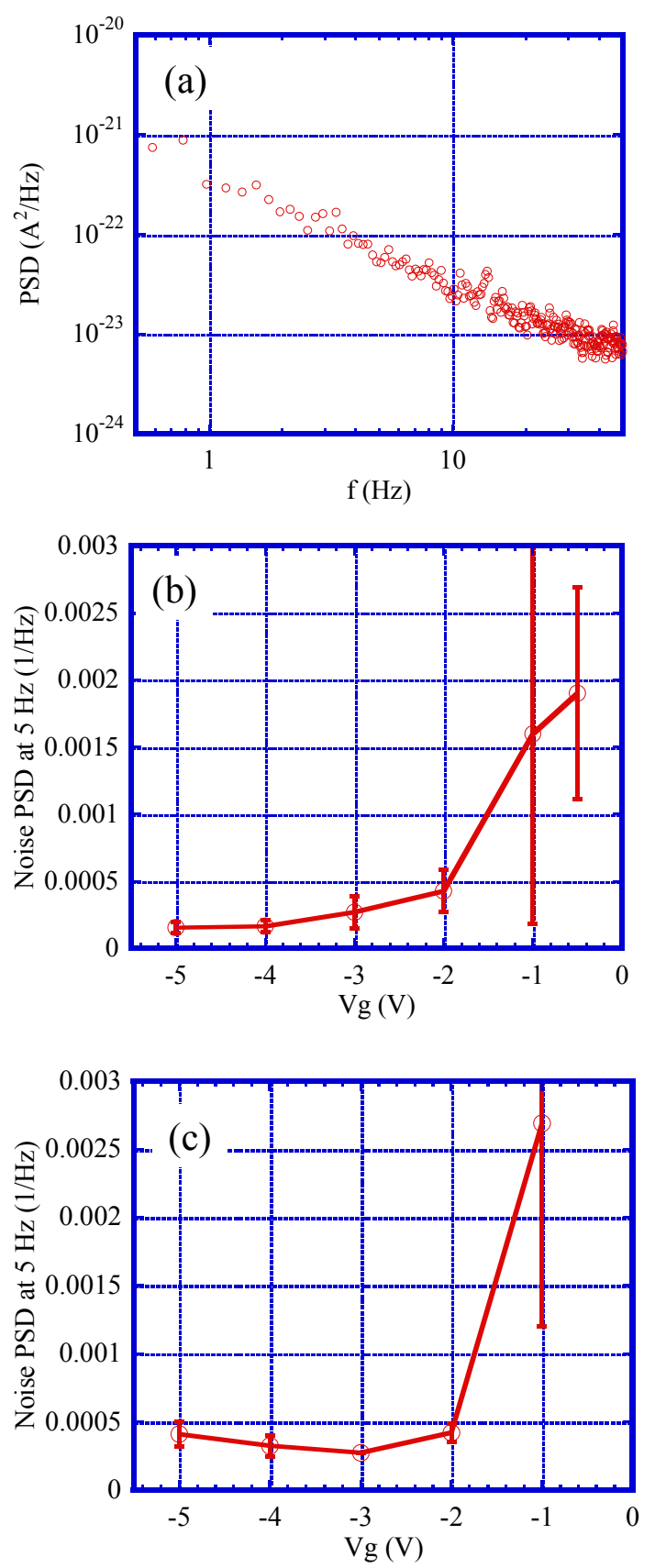

FIG. 6: Noise properties for noise CNTFETs. (a) Spectral density (PSD) for sample 1 , which shows $1 / f$ type noise. $V_{\mathrm{g}}$ is set as $-2 \mathrm{~V}$. $V_{\mathrm{g}}$ dependencies of noise intensity at $5 \mathrm{~Hz}$ for sample 1 (b) and sample 2 (c).

therefore has a constructive role in these model systems. The optimum noise intensity is almost the same for the two systems. Thus, the SR effects can be robustly observed and both noise CNTFETs can be used as noise sources. Therefore, the wider control range for the noise intensity of sample 2 is better as a noise generator for nonlinear systems working with noise.

\section{CONCLUSION}

We have constructed model nonlinear systems using CNTFETs, which seem to be useful as model systems for nano devices. Nano devices should work with noise,
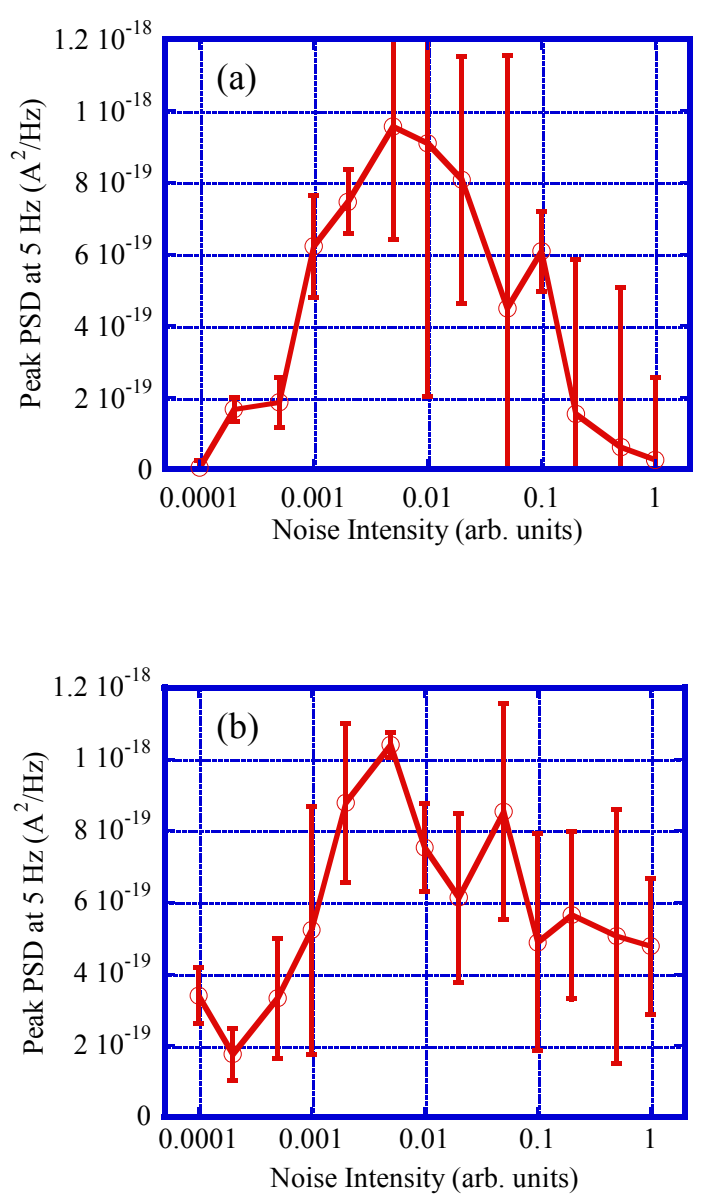

FIG. 7: Noise intensity dependence of PSD for output signals. In (a) and (b), samples 1 and 2 were used for the noise CNTFET, respectively.

and then the constructive roles of noise such as SR are effective for their operation. Therefore, we tried to control the noise from CNTFETs by controlling both $V_{\mathrm{g}}$ and the diameters of CNTs. The size of Co nano particles can be controlled by the deposition amounts using APG. Small amounts of Co catalyst reduce the current of the CNTFET, which could be caused by the small diameter and/or defective CNT channel, including the large contact resistance on the electrodes. This CNTFET has large noise with small carrier number and the controllability by $V_{\mathrm{g}}$ is better, where noise intensity is modified in a wide range by the narrow $V_{\mathrm{g}}$ range. We observed the robustness of the noise enhancements of the response in nonlinear systems. Thus we succeeded in producing controllable model nonlinear systems using CNTFETs.

Further control of CNT devices can achieve $V_{\mathrm{g}}$ control of noise enhancements in a single chip. A more robust SR will also be possible in the near future using the parallel configuration with large current capacity.

\section{Acknowledgments}

The authors acknowledge the generous support from Grant-in-Aid for Scientific Research on Priority Area of the Ministry of Education, Culture, Sports, Science, and Technology (MEXT), and Scientific Research from the Japan Society for the Promotion of Science, and 
Special Education and Research Expenses (Post-Silicon Materials and Devices Research Alliance) from MEXT, Japan. We also acknowledge the support from Future Energy Research Association. Part of this work was supported by "Nanotechnology Network Project of the MEXT, Japan" at Osaka University (Handai Multi- functional Nano-Foundry).

We thank Mr. Yoshiaki Agawa of ULVAC-RIKO, Inc. for discussion concerning Co deposition conditions. We would also like to thank Dr. Shin Mizutani of NTT Communication Science Laboratories.
[1] S. Iijima and T. Ichihashi, Nature 363, 603 (1993).

[2] M. S. Dresselhaus, G. Dresselhaus, and P. C. Eklund, Science of Fullerenes and Carbon Nanotubes (Academic, New York, 1996).

[3] W. Wongwiriyapan, S. Honda, H. Konishi, T. Mizuta, T. Ikuno, T. Ito, T. Maekawa, K. Suzuki, H. Ishikawa, K. Oura, and M. Katayama, Jpn. J. Appl. Phys. 44, L482 (2005).

[4] K. Maehashi, T. Katsura, K. Matsumoto, K. Kerman, Y. Takamura, and E. Tamiya, Anal. Chem. 79, 782 (2007).

[5] K. Maehashi, K. Matsumoto, Y. Takamura, and E. Tamiya, Electroanalysis 21, 1285 (2009).

[6] D. Kaminishi, H. Ozaki, Y. Ohno, K. Maehashi, K. Inoue, K. Matsumoto, Y. Seri, A. Masuda, and H. Matsumura, Appl. Phys. Lett. 86, 113115 (2005).

[7] K. Maehashi, Y. Ohno, K. Inoue, K. Matsumoto, T. Niki, and H. Matsumura, Appl. Phys. Lett. 92, 183111 (2008).

[8] K. Maehashi, H. Ozaki, Y. Ohno, K. Inoue, K. Matsumoto, S. Seki, and S. Tagawa, Appl. Phys. Lett. 90, 023103 (2007).

[9] Y. Ohno, Y. Asai, K. Maehashi, K. Inoue, and K. Matsumoto, Appl. Phys. Lett. 94, 053112 (2009).

[10] S. Sato, A. Kawabata, M. Nihei, and Y. Awano, Chem. Phys. Lett. 382, 361 (2003).

[11] H. Ajiki and T. Ando, J. Phys. Soc. Jpn. 62, 1255 (1993).

[12] J. Kong, A. M. Cassell, and H. Dai, Chem. Phys. Lett. 292, 567 (1998).

[13] Y. Homma, Y. Kobayashi, T. Ogino, D. Takagi, R. Ito, Y. J. Jung, and P. M. Ajayan, J. Phys. Chem. B 107, 12161 (2003).

[14] H. Yoshida, S. Takeda, T. Uchiyama, H. Kohno, and Y. Homma, Nano Lett. 8, 2082 (2008)

[15] M. Hiramatsu, H. Nagao, M. Taniguchi, H. Amano, Y. Ando, and M. Hori, Jpn. J. Appl. Phys. 44, L693 (2005).

[16] L. Gammaitoni, P. Hänggi, P. Jung, and F. Marchesoni, Rev. Mod. Phys. 70, 223 (1998).

[17] C. Nicolis, J. Stat. Phys. 70, 3 (1993).

[18] P. Hänggi, ChemPhysChem 3, 285 (2002).
[19] K. Wiesenfeld and F. Moss, Nature 373, 33 (1995).

[20] I. Goychuk and P. Hänggi, Phys. Rev. E 61, 4272 (2000).

[21] K. Kitajo, D. Nozaki, L. M. Ward, and Y. Yamamoto, Phys. Rev. Lett. 90, 218103 (2003).

[22] I. Y. Lee, X. Liu, B. Kosko, and C. Zhou, Nano Lett. 3, 1683 (2003).

[23] S. Kasai and T. Asai, Appl. Phys. Express 1, 083001 (2008).

[24] P. G. Collins, M. S. Fuhrer, and A. Zettl. Appl. Phys. Lett. 76, 894 (2000).

[25] Y. M. Lin, J. Appenzeller, J. Knoch, Z. Chen, and P. Avouris, Nano Lett. 6, 930 (2006).

[26] K. Murali, S. Sinha, W. L. Ditto, and A. R. Bulsara, Phys. Rev. Lett. 102, 104101 (2009).

[27] D. Tobias, M. Ishigami, A. Tselev, P. Barbara, E. D. Williams, C. J. Lobb, and M. S. Fuhrer, Phys. Rev. B 77, 033407 (2008).

[28] F. N. Hooge, Phys. Lett. A 29, 139 (1969).

[29] Y. Yamamoto, Y. Agawa, Y. Hara, S. Amsno, A. Chayahara, Y. Horino, and K. Fujii, Proc. 12th Int. Ion Implantation Technology, 1148 (1998).

[30] Y. Agawa, K. Yamaguchi, Y. Hara, S. Amano, T. Horiuchi, and G. Shen, ULVAC Tech. J. 57E, 1 (2003).

[31] J. J. Collins, C. C. Chow, and T. T. Imhoff, Phys. Rev. E 52 R3321 (1995).

[32] J. J. Collins, C. C. Chow, and T. T. Imhoff, Nature 376, 236 (1995).

[33] A. Wehrl, Rep. Math. Phys. 30, 119 (1991).

[34] A. Neiman, B. Shulgin, V. Anishchenko, W. Ebeling, L. Schimansky-Geier, and J. Freund, Phys. Rev. Lett. 76, 4299 (1996).

[35] I. Goychuk, Phys. Rev. E 64, 021909 (2001).

[36] M. I. Dykman, D. G. Luchinsky, R. Mannella, P. V. E. McClintock, H. E. Short, N. D. Stein, and N. G. Stocks, Phys. Lett. A 193, 61 (1994).

[37] L. Gammaitoni, Phys. Rev. E 52, 4691 (1995). 